\title{
Roles of Molecular Characteristics in Blood Anticoagulant Activity and Acute Toxicity of Sodium Cellulose Sulfate
}

\author{
Kenji Kamide, Kunihiko OKaJIMA, Toshihiko MATSUI, \\ Masanao OHNISHI* and Hidehiko KOBAYASH ${ }^{* *}$ \\ Textile Research Laboratory, Asahi Chemical Industry Co. Ltd., \\ 11-7 Hacchonawate, Takatsuki, Osaka 569, Japan \\ *Ohnishi Hospital, 4-7-28 Suwa, Joto-ku, Osaka 536, Japan \\ **Development and Technology Administration, \\ Asahi Chemical Industry Co. Ltd., \\ 1-1-2 Yurakucho, Chiyoda-ku, Tokyo 100, Japan
}

(Received November 22, 1982)

\begin{abstract}
An attempt was made to establish correlations among molecular characteristics, blood anticoagulant activity, and acute toxicity of sodium cellulose sulfate (NaCS). For this purpose, $25 \mathrm{NaCS}$ samples ranging in number-average molecular weights $M_{n}$ from 800 to $36.8 \times 10^{4}$ and in total degree of substitutions $\langle\mathrm{F}\rangle$ from 0.5 to 2.75 were prepared. The distribution of substituent groups on three carbon positions $\left(\mathrm{C}_{2}, \mathrm{C}_{3}\right.$, and $\left.\mathrm{C}_{6}\right)$ in a glucopyranose unit $\left\langle\mathrm{f}_{k}\right\rangle(k=2,3$, and 6) of $\mathrm{NaCS}$ was determined by the NMR method. The anticoagulant activity of NaCS to the whole blood was evaluated by the methods of Lee-White, Imai, and the Commentary of Japanese Pharmacopoeia. Acute toxicity, as measured by $\mathrm{LD}_{50}$ (vein injection), of $\mathrm{NaCS}$ for rats was determined by the Probit method. Inhibitory action of NaCS on blood coagulation was estimated using a coagulation factor deficient substrate plasma and a sample plasma from normal blood. It was found that the sum $\left.\left\langle\mathrm{f}_{2}\right\rangle\right\rangle+\left\langle\mathrm{f}_{3}\right\rangle$ is a predominant factor for the anticoagulant activity of NaCS, but $M_{n}$ is only a minor factor. $\mathrm{LD}_{50}$ of $\mathrm{NaCS}$ was found to be intricatedly influenced by $\left\langle\mathrm{f}_{2}\right\rangle+\left\langle\mathrm{f}_{3}\right\rangle$ and $M_{n}$. The inhibitory action of NaCS towards coagulation factor VIII was found to be much more intensive than heparin. An anatomical study showed that after injection of $\mathrm{NaCS}$, no abnormality occurred in live rats but a state of anemia appeared in the liver of dead rats and congestive and blood shots occurred in their lungs.
\end{abstract}

KEY WORDS Sodium Cellulose Sulfate / Total Degree of Substitution / Number-Average Molecular Weight / Anticoagulant Activity / Acute Toxicity / Polymer Drug /

The chemical structure of cellulose sulfate (CS) resembles those of muco-polysaccharides, such as heparin and condroitin sulfate, which are now in wide use as natural-occurring blood anticoagulants. Figure 1 shows (a) three possible positions of the sulfate group in the glucopyranose unit of sodium cellulose sulfate (NaCS) and (b) the chemical structure of the uronic linkage in heparin. $\mathrm{NaCS}$ is linked together by $1,4-\beta$-glucoside, but heparin by $1,4-\alpha-$ glucoside. An anticoagulant activity of $\mathrm{NaCS}$ was first reported by Bergström ${ }^{1}$ as early as 1935 , and thereafter the Biological Institute of Carlsberg Fundation in Copenhagen demonstrated that the coagulation time of whole blood increased by the addition of cellulose trisulfate and other polysaccharide sulfates such as amylose sulfate and amylopectin sulfate. ${ }^{2-6}$ Felling and Wiley, and Rothschild and Castania found many interesting pharmaceutical characteristics of $\mathrm{NaCS}$ including 1) inhibitory action to pancreatic ribonuclease, ${ }^{7}$ 2) kininogen depleting action, ${ }^{8} 3$ ) endo-toxin shock of dogs by the treatment of $\mathrm{NaCS} .{ }^{9}$ The aminosulfate group at the $\mathrm{C}_{2}$ position of uronic units in heparin was found to play an important role in anticoagulant activity. ${ }^{10}$ Desulfation of this aminosulfate group was found to lower the anticoagulant activity of heparin. These experimetal results on heparin suggest strongly that the physiological 
a)

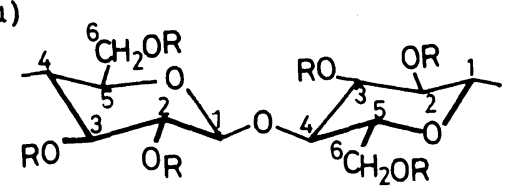

$\mathrm{R}: \mathrm{SO}_{3} \mathrm{Na}$ or $\mathrm{H}$

b)

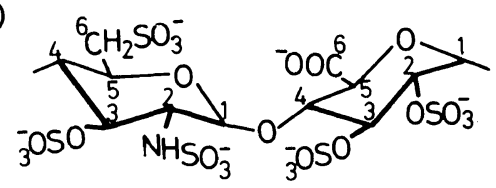

Figure 1. Chemical structure of sodium cellulose sulfate (a) and heparin (b). Here, the $\mathrm{C} 1$ chair conformation is assumed for both compounds.

activity, including anticoagulant activity, of polymers is closely related to their molecular characteristics. Thus, we may expect for $\mathrm{NaCS}$ that its anticoagulant activity and other pharmaceutical activities are influenced by molecular parameters such as molecular weight, chain structure, distribution of substituent groups along the molecular chain, the probability of substitution at $\mathrm{C}_{2}, \mathrm{C}_{3}$ and $\mathrm{C}_{6}$ positions of glucopyranose units $\left(\left\langle\mathrm{f}_{k}\right\rangle, k=2,3\right.$ and 6), and the total degree of substitution $\langle\mathrm{F}\rangle$. Unfortunately knowledge of the molecular characteristics of $\mathrm{NaCS}$ is as yet in a very primitive state because of its less practical importance and the experimental difficulty associated with its polyelectrolytic nature.

We recently succeeded, by nuclear magnetic resonance (NMR), in determining the distribution of sulfate groups in glucopyranose units of $\mathrm{NaCS}^{11}$ and also evaluated, by viscometry, membrane osmometry and light scattering, the dilute solution properties of this polymer. ${ }^{12}$ This article attempts to find a correlation of molecular characteristics of $\mathrm{NaCS}$ with its anticoagulant activity and acute toxicity.

\section{EXPERIMENTAL}

\section{Synthesis of Sodium Cellulose Sulfate (NaCS)}

25 samples of sodium cellulose sulfate $(\mathrm{NaCS})$ having number-average molecular weight $M_{n}=$ $800-36.8 \times 10^{4}$ and total degree of substituent $\langle\mathrm{F}\rangle=0.50-2.75$ were synthesized as follows: 20 gram ( $\mathrm{g}$ ) of acid hydrolyzed wood pulp (viscosity- average degree of polymerization $=50-3000$ ) in the form of sheet or flock was allowed to absorb $40 \mathrm{ml}$ of dimethylformamide (DMF). The DMF-wetted pulp thus prepared was mixed at room temperature with a given amount (86-130 g) of DMF/sulfur trioxide $\left(\mathrm{SO}_{3}\right)$ complex $(2: 1, \mathrm{~mol} / \mathrm{mol})$ precooled at $0^{\circ} \mathrm{C}$. The mixture was stirred vigorously at $25^{\circ} \mathrm{C}$ for $4 \mathrm{~h}$. The resultant solution was diluted with a large volume of water, immediately neutralized with $2 \mathrm{~N}$ sodium hydroxide $(\mathrm{NaOH})$, and methanol was added to it to precipitate the crude $\mathrm{NaCS}$. The dried crude $\mathrm{NaCS}$ was dissolved in distilled water and the resulting brownish solution was passed through a diatomaceous earth layer to remove any impurities, and the polymer was reprecipitated with methanol. The polymer was washed several times with a mixture of methanol/water $(7: 3, \mathrm{v} / \mathrm{v})$ and again dissolved in deionized water and finally subjected to dialysis against deionized water until the electroconductivity of the dialyzing water became below $10^{-5}$ $\mathrm{MS} / \mathrm{cm}$. Pure NaCS obtained by the evaporation of the solvent was designated as CS. By this method, $16 \mathrm{NaCS}$ samples with $\langle\mathrm{F}\rangle=1.36-2.75$ were prepared. Of these samples, CS-11 was resulfated under the same conditions used above and designated CSD. This had $\langle F\rangle=1.99$, which was almost the same as that of CS-11, but had a different $\left\langle\mathrm{f}_{k}\right\rangle$. For example, $\left\langle\mathrm{f}_{6}\right\rangle$ changed from 0.3 to 0.67 by resulfation (see Table I). In order to lower $\langle\mathrm{F}\rangle$, the above synthetic procedure was modified so that the solution of $\mathrm{NaCS}$ in $\mathrm{DMF} / \mathrm{SO}_{3}$ was heated at $70^{\circ} \mathrm{C}$ for $30 \mathrm{~min}$ before neutralization. This procedure gave $\mathrm{NaCS}$ having $\langle\mathrm{F}\rangle=1.05$, and the product was coded as DSH. A NaCS sample coded as CS-A was obtained from a pulp depolymerized by repeated acid- and alkali-hydrolysis, using the same conditions as in preparing CS samples. NaCS with low 《F》 was prepared by the following procedure: Acid-hydrolyzed pulp was immersed in a large volume of a mixture of $98 \%$ sulfuric acid $\left(\mathrm{H}_{2} \mathrm{SO}_{4}\right)$ / $n$-butanol $(110: 29, \mathrm{v} / \mathrm{v})$ at $5-10^{\circ} \mathrm{C}$ for $10 \mathrm{~min}$ and the excess mixture was squeezed out of the pulp by hands. The resulting gelatineous liquid was maintained at $0-5^{\circ} \mathrm{C}$, then partially neutralized with $30 \%$ aqueous $\mathrm{NaOH}$, and crude CS was precipitated by adding diethylether containing dry carbon dioxide. The viscous crude CS was again neutralized with $5 \%$ aqueous $\mathrm{NaOH}$ and precipitated with methanol. The precipitated $\mathrm{NaCS}$ was purified by the same method as in the $\mathrm{DMF} / \mathrm{SO}_{3}$ complex 


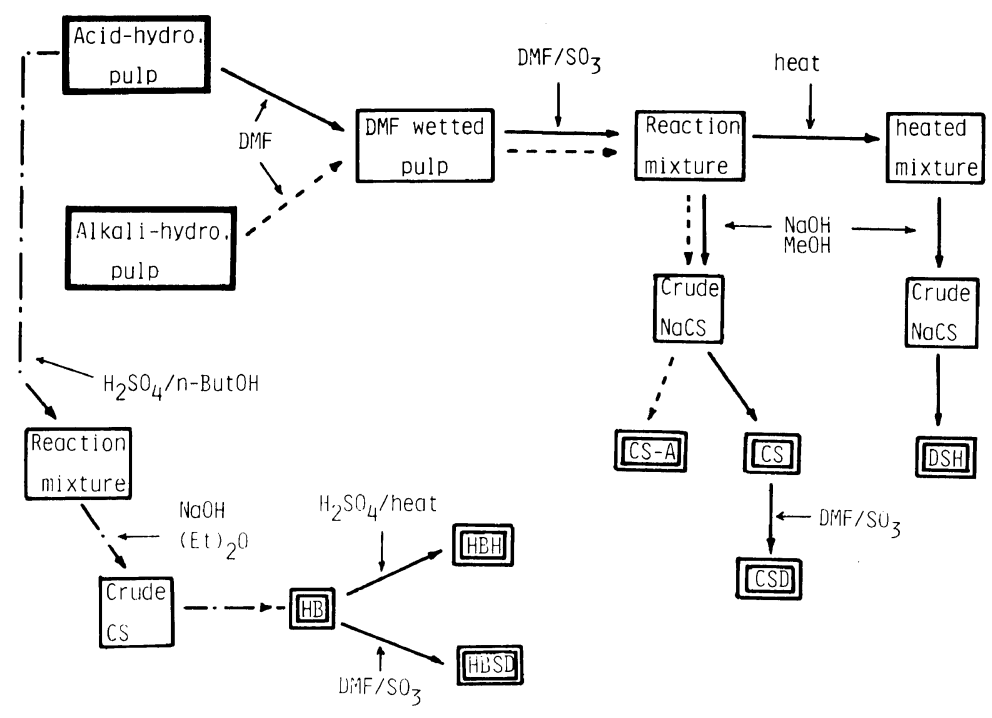

Figure 2. Schematic representation of the synthesis procedures of NaCS. Double rectangles indicate sample codes (see the text).

method, and coded HB. Four HB samples obtained had $\langle\mathrm{F}\rangle$ of $0.7-0.95$. NaCS coded as HBSD was obtained by resulfating sample HB-1 using the $\mathrm{DMF} / \mathrm{SO}_{3}$ complex. $\langle\mathrm{F}\rangle$ of $\mathrm{HBSD}$ was the same as that for HB-1 $(\langle\mathrm{F}\rangle=0.95)$, but the $\left\langle\mathrm{f}_{k}\right\rangle$ was different. NaCS with extremely low $M_{n}$, coded as $\mathrm{HBH}$, was obtained by heating sample $\mathrm{HB}-1$ in $6 \mathrm{~N}$ aqueous $\mathrm{H}_{2} \mathrm{SO}_{4}$ at $70^{\circ} \mathrm{C}$ for $1 \mathrm{~h}$. $\langle\mathrm{F}\rangle$ of $\mathrm{HBH}$ was 0.5 , lower than that of HB-1. All synthetic procedures used in this study are summerized in Figure 2.

A commercial sample of heparin sodium salt, which had an anticoagulant activity $\chi$ of $152 \mathrm{IU} / \mathrm{mg}$ as measured by Japanese Pharmacopoeia, was obtained from Nakarai Chemicals Co. Ltd. (Kyoto). A purified heparin with $\chi$ of $188-228$ was prepared by treating this commercial heparin with ethanol.

\section{Molecular Weight of $\mathrm{NaCS}$}

The $M_{n}$ of the NaCS samples were determined with a Melab membrane osmometer model CMS-1 and a Hewlett-Packard high-speed membrane osmometer model 502 at $25^{\circ} \mathrm{C}$ with $0.5 \mathrm{M}$ aqueous $\mathrm{NaCl}$ as a solvent. ${ }^{12}$ Selecton B 19 (made by Schleiche \& Schnell Inc.) was used as the membrane.
$《 F \gg$ and the Distribution of Sulfate Groups in Glucopyranose Units

The total degree of substitution, $\langle\mathrm{F}\rangle$ weightaveraged over all glucopyranose units, was determined gravimetrically according to the method described by Schweiger ${ }^{13}$ and denoted as $\langle\mathrm{F}\rangle_{\mathrm{g}}$. The amount of sulfric acid liberated by boiling $\mathrm{NaCS}$ in $1 N$ hydrochloric acid was taken as that of barium sulfate. The glucopyranose unit constituting a cellulose molecule has one primary hydroxyl group at $\mathrm{C}_{6}$ and two secondary hydroxyl groups at $\mathrm{C}_{2}$ and $\mathrm{C}_{3}$ (see Figure 1). The degree of sulfate substitution is different at different $\mathrm{C}$ positions, but its average over the three $\mathrm{C}$ positions is essential for characterizing $\mathrm{NaCS}$. We denoted the average probability of hydroxyl group substitution at the $\mathrm{C}_{k}(k=2,3$ and 6) position by $\left\langle\mathrm{f}_{k}\right\rangle$ and determined it for 13 NaCS samples, using the ${ }^{1} \mathrm{H}$ NMR method proposed by Kamide and Okajima. ${ }^{11}$ The sum of $\left\langle\mathrm{f}_{2}\right\rangle$, $\left\langle\mathrm{f}_{3}\right\rangle$ and $\left\langle\mathrm{f}_{6}\right\rangle$ is denoted by $\langle\mathrm{F}\rangle_{\mathrm{NMR}}$.

\section{Evaluation of the Anticoagulant Activity of $\mathrm{NaCS}$}

Method of Lee-White. Anticoagulant activity $\left(A_{\mathrm{LW}}\right)$ was evaluated by a modification of the method of Lee-White ${ }^{14}$ in a vessel thermostated at $37^{\circ} \mathrm{C}$. The modification was made as follows. The inner wall of a polyethylene injector (inner diameter $1.0 \mathrm{~cm}$, volume $2.5 \mathrm{ml}$, manufactured by Nippro 
Co.) was first washed with physiological saline. $1.0 \mathrm{ml}$ of physiological saline dissolving an anticoagulant at a concentration of $0.1 \mathrm{wt} \%$ was sucked into the injector and the anticoagulant solution was pressed out by hand. It was expected that a trace of anticoagulant remained adhering to the inner wall of the injector. $0.1 \mathrm{ml}$ of fresh human whole blood was admitted into the injector and $0.1 \mathrm{ml}$ of air was sucked into the injector. During this operation, the injector was maintained in an upright position. We measured the time $t_{1}$ necessary for the test blood to start forming small coagulated blood gel particles by observing the blood flow pattern on the injector wall while the injector was gently being shaken. We also measured the time $t_{2}$ required for completion of blood coagulation (i.e., clotting or thrombi), as usually done in measuring anticoagulant activity. These two times $t_{1}$ and $t_{2}$ were found to be closely correlated to each other. After the completely coagulated blood gel was removed by placing the injector upside down, the amount of blood $m_{1}$ remaining on the wall was roughly estimated. By comparing the results with those of heparin, the anticoagulant activity $A_{\mathrm{Lw}}$ of NaCS estimated by the Lee-White method was classified into four grades: superior (S), good (G), inferior (I), and non-active $(\mathrm{N})$.

Method of Imai ${ }^{15}$. The amount of formaldehydefixed thromb was determined at $37^{\circ} \mathrm{C}$. A watch glass of $11 \mathrm{~cm}$ in diameter was first washed with physiological saline, wiped with pure cotton gauze, and then $0.02 \mathrm{ml}$ of physiological saline containing $1.0 \mathrm{wt} \%$ anticoagulant was dropped onto the center of the watch glass and spread by swirling action. $0.30 \mathrm{ml}$ of the fresh human whole blood was placed on this glass, made to stand for a given time, fixed with $37 \%$ formaldehyde, washed with water, dried in air, and the weight of thromb formed, $m_{2}$, was measured with a precision balance.

Method According to the Commentary of Japanese Pharmacopoeia. The method described in the Commentary of Japanese Pharmacopoeia ${ }^{16}$ provides another parameter of anticoagulant activity, $\chi$, which is expressed in international units (IU)/mg of heparin. For the evaluation of $\chi$ for NaCS, the original procedure was slightly modified as follows:

(1) Prepare solutions of different concentrations by dissolving a heparin standard with $\chi$ of 152 $\mathrm{IU} / \mathrm{mg}$ (hereafter denoted as $\chi_{0}$ ) or $\mathrm{NaCS}$ in physiological saline.
(2) Add $0.02 \mathrm{ml}$ of these solutions to $1.0 \mathrm{ml}$ of the fresh human whole blood $\left(\mathrm{B}^{-}\right.$, male adult) and measure the coagulation time $\left(t_{3}\right)$ in second. Measurements are made at different concentrations $\left(0-75 \times 10^{-3} \mathrm{mg} \mathrm{ml}^{-1}\right)$ of the heparin standard and the polymer sample.

(3) For each solution, plot $\log t_{3}$ against the absolute amount $m_{3}$ (in $\mathrm{mg}$ ) of the anticoagulant used.

(4) Determine the slopes of the plots for both the standard and the polymer sample.

(5) Calculate $\chi(\mathrm{IU} / \mathrm{mg})$ of the polymer sample from

$$
\chi=\chi_{0} \times \frac{\mathrm{d} \log t_{3} / \mathrm{d} m_{3}}{\left(\mathrm{~d} \log t_{3} / \mathrm{d} m_{3}\right)_{0}}
$$

where the subscript 0 denotes the heparin standard. The plot in step (4) was found to give a straight line.

\section{Physiological Action of NaCS to Various Co- agulation Factors in Blood}

All assays of the physiological action of $\mathrm{NaCS}$ to coagulation factors were made at $37^{\circ} \mathrm{C}$ with heparin as the reference. We used specially treated plasma, in which one of the coagulation factors was deficient

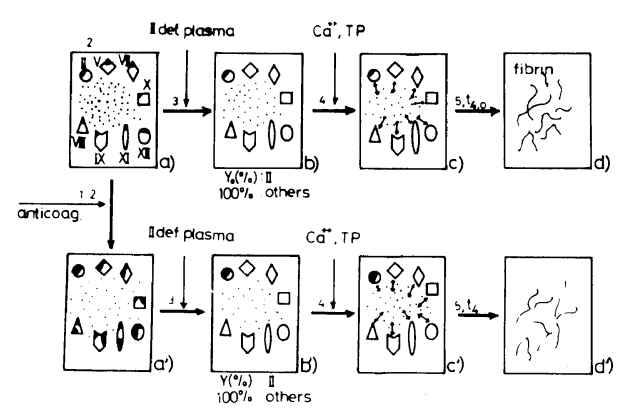

Figure 3. Scheme of the principle for measuring the existence ratio $Y$ of coagulation factor II: Numbers on the figure denote the steps described in the text.

(a) sample plasma from normal human whole blood. Shaded area indicates $\left(100-Y_{0}\right)(\%)$.

(b) sample plasma containing a coagulation factor II deficient substrate plasma. $Y$ of all factors, other than that of factor II gives $100 \%$ when added.

(c) Fibrinogen in (b) was activated by addition of activated thromboplastin.

(d) Coagulated plasma in which fibrin was formed.

(a') sample plasma containing anticoagulant $\mathrm{NaCS}$. Shaded area indicates $\left(100-Y_{0}\right) \%$ and black area $\left(Y_{0}-Y\right) \%$.

$\left(b^{\prime}\right)-\left(d^{\prime}\right)$ equals (b) - (d); dots denote fibrinogen. 
(hereafter referred to as a coagulation factor deficient substrate plasma) and evaluated the percentage of the coagulation factors existing in a given sample plasma. For this purpose, the conventional procedure was modified by adding in advance an anticoagulant to the sample plasma. The procedure scheme is given in Figure 3. The details are as follows:

a) Determination of the Existence Ratios $Y$ of Internal Coagulation Factors II, V, VII, and X.

(1) Put $0.01 \mathrm{ml}$ of physiological saline solution containing $2 \times 10^{-4} \mathrm{mg}$ of heparin or $1.6 \times$ $10^{-4} \mathrm{mg}$ of $\mathrm{NaCS}$ in a test tube having an inner diameter of $0.8 \mathrm{~cm}$ and maintained at $37^{\circ} \mathrm{C}$.

(2) Dilute one volume of the sample plasma with nine volumes of an Olenveronal buffer solution, supplied by Diagnostic Inc., USA, and put $0.1 \mathrm{ml}$ of this solution in the above-mentioned tube and leave the system at $37^{\circ} \mathrm{C}$.

(3) Prepare four kinds of coagulation factor deficient plasmas (deficient in II, V, VII, and X, respectively) (Diagnostic Inc., USA). Add $0.1 \mathrm{ml}$ of the plasma to $0.1 \mathrm{ml}$ of the solution in step (1) and allow the tube to stand for $2 \mathrm{~min}$.

(4) Blow $0.1 \mathrm{ml}$ of an activated thrombo-plastin/ calcium chloride mixture (Diagnostic Inc., USA) into the tube in step (3).

(5) Measure the time $\left(t_{4}\right)$ necessary for fibrin to start growing.

(6) Calculate the existence ratios $Y(\%)$ of individual coagulation factors using the following equations, derived from the experimental relationships supplied in the form of graphs by Diagnostic Inc., USA. ${ }^{17}$

$$
\begin{aligned}
\log t_{4}=- & 0.19 \log Y+1.475 \\
& \text { for factor II } \\
\log t_{4}=- & 0.30 \log Y+1.879 \\
& \text { for factor V } \\
\log t_{4}=- & 1.445 \log Y+1.742 \\
& \text { for factor VII } \\
\log t_{4}=- & 0.2824 \log Y+1.799
\end{aligned}
$$$$
\text { for factor } \mathrm{X}
$$

b) Determination of the Existence Ratios $Y$ of External Coagulation Factors VIII, IX, XI, and XII. For this purpose, we slightly modified the steps for the determination of internal coagulation factors as follows. In step (2), one volume of the sample plasma was diluted with four volumes of the Olenveronal buffer, and in step (4), a cepharoplastin/calcium chloride mixture was used in place of the thromboplastin/calcium chloride mixture. The values of $Y$ of the external coagulation factors were calculated from the experimental relations:

$$
\begin{aligned}
\log t_{4}=- & 0.1709 \log Y+2.0325 \\
& \text { for factor VII } \\
\log t_{4}=- & 0.1776 \log Y+2.0363 \\
& \text { for factor IX } \\
\log t_{4}=- & 0.250 \log Y+2.1236 \\
& \text { for factor XI } \\
\log t_{4}=- & 0.2189 \log Y+2.22147
\end{aligned}
$$$$
\text { for factor XII }
$$

In the procedures described in a) and b), some loss may occur in the activity of the coagulation factors when the sample plasma and the anticoagulant are mixed. However, it is assumed here that when a coagulation factor deficient substrate plasma is added to the mixture of normal plasma and an anticoagulant the activities of all coagulation factors other than the deficient coagulation factor recovered to $100 \%$. The fundamental concept underlying the method for determining $Y$ is illustrated in Figure 3. Since the values of $Y$ for various coagulation factors in the sample plasma were determined after adding the anticoagulant, the ability of an anticoagulant to inactivate the coagulation factor under consideration can be defined by $100\left(Y_{0}-Y\right) / Y_{0}$, where $Y_{0}$ is the value of $Y$ when no anticoagulant is added. It should be noted that here $Y$ was obtained only at one dose level $\left(2 \times 10^{-4} \mathrm{mg}\right)$. Linear relations between the logarithmic coagulation time $t_{4}$ (expressed in second) and the absolute amount $m_{4}$ of anticoagulant were confirmed at least within the concentration range of the anticoagulant studied. Thus, the slope of the plot of $\log t_{4} v s . m_{4}$ could be taken as another measure of the inhibitory action of the anticoagulant toward the coagulation factor. 


\section{$L D_{50}$ When Anticoagulant is Injected Into the Vein of a Rat}

Four-weeks-old male rats weighing $21-23 \mathrm{~g}$ were used. The rats were confined to shaving beds in a plastic cage and allowed to eat and drink at will. The cage was changed every $3-4$ days. An NaCS solution (concentration $0.25-0.8 \mathrm{wt} \%$ ) in physiological saline was injected into a vein at a rate of $0.1 \mathrm{ml} / 10 \mathrm{~s}$, and general symptoms and changes in body-weight were observed during the following three weeks, using $10-20$ rats for a given dose level. $\mathrm{LD}_{50}$ was determined from the death ratio one week after the injection, according to the Canola-SX-50 (Probit) method. ${ }^{18}$ Both dead and live rats during the test were subjected to anatomy and intestinal abnormalities were visually examined.

\section{RESULTS AND DISCUSSION.}

Figure 4 shows some typical ${ }^{1} \mathrm{H}$ NMR spectra of $\mathrm{NaCS}$ in deuterium oxide. Figures $4 \mathrm{a}$ ) and b) refer to two NaCS samples having nearly the same $\langle\mathrm{F}\rangle$ (1.96-1.97), but different $\left\langle\left\langle\mathrm{f}_{k}\right\rangle .\langle\mathrm{F}\rangle\right.$ decreased in the order: $a) \simeq b$ ) $>$ c) $>$ d). The NMR signal for the proton at the $\mathrm{C}_{1}$ position shifts very slightly to higher magnetic field as $\langle F\rangle$ decreases. The structure of the spectrum in the range from 3.4 to $4.0 \mathrm{ppm}$ becomes complicated with a decrease in $\langle\mathrm{F}\rangle$, approaching that for pure cellulose.

Table I lists the values of $M_{n},\langle\mathrm{~F}\rangle$, and the anticoagulant parameters $A_{\mathrm{LW}}, m_{2}$ and $\chi$, and $\mathrm{LD}_{50}$ of $25 \mathrm{NaCS}$ samples and heparin. For the selected $13 \mathrm{NaCS}$ samples, the distribution of sulfate groups over the three possible positions in a glucopyranose unit $\left(\left\langle\mathrm{f}_{k}\right\rangle(k=2,3\right.$ and 6$\left.)\right)$ is also given in this table. It can be seen that $\langle\vec{F}\rangle_{\mathrm{NMR}}$ agrees with $\langle\mathrm{F}\rangle_{\mathrm{g}}$ within an uncertainty of \pm 0.10 . The correlation coefficient between these was estimated to be 0.998 . In a previous study ${ }^{12}$ it was found that the ratio of weight- to number-average molecular weight $M_{w} / M_{n}$ of CS samples ranged from 3.1 to 4.1 . The procedures employed here for synthesis of $\mathrm{NaCS}$ afforded samples having a great variety of $M_{n}$ and $《 \mathrm{~F}\rangle$. Thus, the $M_{n}$ of these sample varied from 800 to $36.8 \times 10^{4}$ and their $\langle\mathrm{F}\rangle$ from 0.5 to 2.75 .

Figure 5 shows the relation between $A_{\mathrm{LW}}$ and $m_{2}$ or $\chi . A_{\mathrm{LW}}$ of the $\mathrm{S}$ grade corresponds to $m_{2}$ of 0.4 2.8 and $\chi$ of $188-246$ and $A_{\mathrm{LW}}$ of the grade $\mathrm{G}$ to $m_{2}$ of $4.8-5.3$ and $\chi$ of $152-206$. $A_{\mathrm{LW}}$ of the I grade ranges in $m_{2}$ from 12.6 to 26.3 and in $\chi$ from 58 to

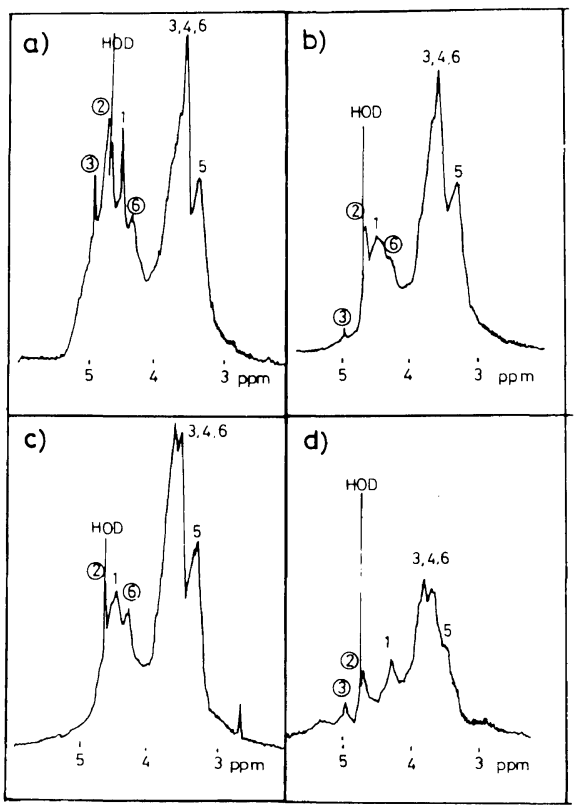

Figure 4. Typical ${ }^{1} \mathrm{H}$ NMR spectra of $\mathrm{NaCS}$ samples in deuterium oxide: a) CS-A; b) CS-11; c) HBSD; d) $\mathrm{HBH}$. Numbers indicate the position of carbon atoms (see Figure 1) and those in the circles denote the position of carbon atoms linking the hydroxyl residues.

124 and $A_{\mathrm{LW}}$ of the $\mathrm{N}$ grade corresponds to $m_{2}$ larger than 42 and $\chi$ lower than 15 . Some overlaps and gaps in $m_{2}$ and $\chi$ for different grades exist because of the qualitative nature of $A_{\mathrm{Lw}}$ and also because of the insufficient number of polymer samples. It is of interest to see that $\mathrm{NaCS}$ has a wide range of anticoagulant activity.

Figure 6 shows the relation between $A_{\mathrm{LW}}$ and 《F》 of NaCS. Qualitatively, $A_{\mathrm{LW}}$ has a tendency to approach the $\mathrm{G}$ or $\mathrm{S}$ grade with an increase in $\langle\mathrm{F}\rangle$. In particular, for samples with $\langle\mathrm{F}\rangle$ less than 1.94 , $A_{\mathrm{LW}}$ was of neither $\mathrm{G}$ nor $\mathrm{S}$ grade, and all $\mathrm{HB}$ samples which had $\langle\mathrm{F}\rangle$ less than unity, showed very low anticoagulant activity. $\langle\mathrm{F}\rangle$ is not the only factor controlling $A_{\mathrm{LW}}$, because when $\langle\mathrm{F}\rangle$ is in the range $1.88-2.05, A_{\mathrm{LW}}$ covers all grades varying from the $\mathrm{N}$ to the $\mathrm{S}$. All CS samples having $\langle\mathrm{F}\rangle$ larger than 1.88 showed $A_{\mathrm{LW}}$ of the $\mathrm{S}$ or $\mathrm{G}$ grade, but sample CS-A, which had $\langle\mathrm{F}\rangle$ of 1.97 , showed $A_{\mathrm{Lw}}$ of the $\mathrm{N}$ grade. It should be noted that there is a great difference in $\left\langle\mathrm{f}_{2}\right\rangle+\left\langle\left\langle\mathrm{f}_{3}\right\rangle\right.$ between the CS samples (>1.6) and sample CS-A (0.97). Sample CSD had almost the same $\langle F\rangle(=2.00)$ as sample 
Blood Anticoagulant Activity and $\mathbf{L D}_{50}$ of $\mathrm{NaCS}$

Table I. Characterization of sodium cellulose sulfate

\begin{tabular}{|c|c|c|c|c|c|c|c|c|c|c|}
\hline \multirow[b]{2}{*}{ Sample } & \multirow[b]{2}{*}{$\begin{array}{c}M_{n} \\
\times 10^{-4}\end{array}$} & \multicolumn{2}{|c|}{ Total $《 F\rangle$} & \multicolumn{3}{|c|}{ Distribution of sulfate } & \multicolumn{3}{|c|}{ Anticoagulant activity } & \multirow{2}{*}{$\frac{\mathrm{LD}_{50}}{\mathrm{mg} \mathrm{kg}^{-1} \text {-rat }}$} \\
\hline & & $\begin{array}{l}\text { Gravi- } \\
\text { metry }\end{array}$ & NMR & $\left\langle\mathrm{f}_{2} 》\right.$ & $\left\langle\mathrm{f}_{3}\right\rangle$ & $\left\langle\mathrm{f}_{6}\right\rangle$ & $A_{\mathrm{wL}}$ & $\frac{m_{2}}{\mathrm{mg}}$ & $\frac{\chi}{\mathrm{IU} / \mathrm{mg}}$ & \\
\hline CS-1 & 36.8 & 1.66 & - & - & - & - & $\mathbf{I}^{\mathrm{a}}$ & 26.3 & - & - \\
\hline $\mathrm{CS}-2$ & 9.39 & 1.94 & - & - & - & - & $G^{b}$ & - & - & - \\
\hline DSH & 6.91 & 1.05 & 1.05 & 0.55 & 0.00 & 0.50 & $\mathrm{~N}^{\mathrm{c}}$ & - & 0 & 730 \\
\hline $\mathrm{CS}-3$ & 6.79 & 1.98 & - & - & - & - & $\mathrm{G}$ & - & - & - \\
\hline $\mathrm{CS}-4$ & 6.65 & 2.39 & 2.46 & 1.00 & 0.74 & 0.72 & G & 4.8 & $184-228$ & 53.6 \\
\hline CS-5 & 5.32 & 1.36 & 1.36 & 0.75 & 0.42 & 0.19 & I & - & $55-61$ & 513 \\
\hline CS-6 & 5.31 & 1.84 & - & - & - & - & I & 12.6 & - & - \\
\hline $\mathrm{CS}-7$ & 5.06 & 1.84 & - & - & - & - & I & - & - & - \\
\hline CS-8 & 5.06 & 1.73 & - & - & - & - & I & - & - & - \\
\hline CS-9 & 4.98 & 1.81 & - & - & - & - & I & - & - & - \\
\hline CS-10 & 4.32 & 2.75 & 2.75 & 1.00 & 0.92 & 0.83 & $S^{d}$ & 0.8 & $230-242$ & 25.2 \\
\hline CS-A & 3.15 & 1.97 & 1.97 & 0.71 & 0.26 & 1.00 & $\mathrm{~N}$ & 42.0 & $7-8$ & 5000 \\
\hline CS-11 & 2.48 & 1.98 & 1.96 & 1.00 & 0.61 & 0.34 & $\mathrm{~S}$ & 2.8 & $175-219$ & 43 \\
\hline HB-1 & 2.26 & 0.95 & 0.95 & 0.52 & 0.00 & 0.43 & $\mathrm{~N}$ & 46.3 & 0 & $4634-5326$ \\
\hline CS-12 & 1.95 & 2.03 & 1.93 & 1.00 & 0.65 & 0.28 & $\mathrm{~S}$ & 1.2 & $200-222$ & 63.8 \\
\hline CS-13 & 1.80 & 1.74 & - & - & - & - & I & - & - & - \\
\hline CS-14 & 1.70 & 2.60 & 2.62 & 1.00 & 0.87 & 0.85 & $\mathrm{~S}$ & 0.4 & $242-251$ & 38.3 \\
\hline CSD & 1.69 & 2.05 & 1.99 & 0.75 & 0.55 & 0.67 & I & - & $117-130$ & 726 \\
\hline CS-15 & 1.57 & 1.96 & - & - & - & - & $S$ & 0.6 & - & - \\
\hline CS-16 & 1.57 & 1.94 & 1.97 & 1.00 & 0.60 & 0.37 & $\mathrm{~S}$ & 0.6 & $167-209$ & 184.5 \\
\hline HB-2 & 1.24 & 0.70 & - & - & - & - & I & 18.5 & - & - \\
\hline HB-3 & 1.16 & 0.74 & - & - & - & - & I & 13.7 & - & - \\
\hline HBSD & 0.99 & 0.95 & 0.95 & 0.29 & 0.33 & 0.33 & $\mathbf{N}$ & - & $10-11$ & 1004 \\
\hline ப்B-4 & 0.92 & 0.76 & - & - & - & - & I & 12.7 & - & - \\
\hline $\mathrm{HBH}$ & 0.08 & 0.50 & 0.58 & 0.31 & 0.27 & 0.00 & $\mathrm{~N}$ & - & $12-15$ & 712 \\
\hline Heparin & - & - & - & - & - & - & G & 5.3 & 152 & 1200 \\
\hline
\end{tabular}

a I denotes inferior.

b $\mathrm{G}$ denotes good equivalent.

c $\mathrm{N}$ denotes non-active.

d $\mathrm{S}$ denotes superior.

CS-11. The most significant difference between these two polymers is that CSD had a lower $\left\langle\left\langle\mathrm{f}_{2}\right\rangle+\left\langle\mathrm{f}_{3}\right\rangle\right.$ (or $\left.\left\langle\mathrm{f}_{2}\right\rangle\right\rangle$ and $\left.\left\langle\mathrm{f}_{3}\right\rangle\right)$, and its $A_{\mathrm{LW}}$ was one grade below that of CS-11. Attempts to increase $\langle\mathrm{F}\rangle$ by resulfation of sample HB-1 with the DMF/ $\mathrm{SO}_{3}$ complex failed, and no improvement of $A_{\mathrm{LW}}$ was obtained in spite of the resulting marked changes in $\left\langle\mathrm{f}_{k}\right\rangle$ (for example, $\left\langle\mathrm{f}_{3}\right\rangle$ changed from zero to 0.34 ). These facts suggest that $A_{\mathrm{LW}}$ is related not only to $\langle\mathrm{F}\rangle$ but also to the average distribution of the substituent groups in glucopyranose units $\left(\left\langle\mathrm{f}_{k}\right\rangle, k=2,3\right.$ and 6$)$.

Figure 7 shows $A_{\mathrm{LW}}$ of $\mathrm{NaCS}$ as a function of $\left\langle\left\langle\mathrm{f}_{2}\right\rangle\right\rangle,\left\langle\mathrm{f}_{3}\right\rangle,\left\langle\left\langle\mathrm{f}_{6}\right\rangle\right.$, and $\left\langle\mathrm{f}_{2}\right\rangle+\left\langle\left\langle\mathrm{f}_{3}\right\rangle,\left\langle\mathrm{f}_{2}\right\rangle,\left\langle\mathrm{f}_{3}\right\rangle\right.$, and $\left\langle\mathrm{f}_{2}\right\rangle+\left\langle\left\langle\mathrm{f}_{3}\right\rangle\right.$ show good correlation with $A_{\mathrm{LW}}$, but $\left\langle\mathrm{f}_{6}\right\rangle$ does not. All NaCS samples with $\left\langle\mathrm{f}_{2}\right\rangle<0.75$ or $\left\langle\mathrm{f}_{3}\right\rangle\left\langle 0.48\right.$ have $A_{\mathrm{LW}}$ of the $\mathrm{N}$ grade (in this case, $\left\langle\mathrm{f}_{2}\right\rangle$ and $\left\langle\mathrm{f}_{3}\right\rangle$ are closely correlated with one another). $\left.\left\langle\mathrm{f}_{2}\right\rangle\right\rangle+\left\langle\mathrm{f}_{3}\right\rangle$ has the best correlation with $A_{\mathrm{LW}}$.

Table I suggests that the average molecular weight $M_{n}$ has a significant effect on $A_{\mathrm{LW}}$. As far as CS samples are concerned, $A_{\mathrm{LW}}$ tends to increase with a decrease in $M_{n}$.

Figure 8 shows the relation between $\chi$ and the distribution of the sulfate groups, represented by $\left.\left(\left\langle\mathrm{f}_{2}\right\rangle\right)+\left\langle\mathrm{f}_{3}\right\rangle\right) / 3, \quad\left\langle\mathrm{f}_{6}\right\rangle / 3$, and $(3-\langle\mathrm{F}\rangle) / 3$. The theoretical maxima of $\left(\left\langle\mathrm{f}_{2}\right\rangle+\left\langle\left\langle\mathrm{f}_{3}\right\rangle\right) / 3,\left\langle\mathrm{f}_{6}\right\rangle / 3\right.$, and $\langle F\rangle$ are $0.67,0.33$, and 3 , respectively. The full lines in the figure indicate the contours of the same $\chi \cdot \chi$ is 


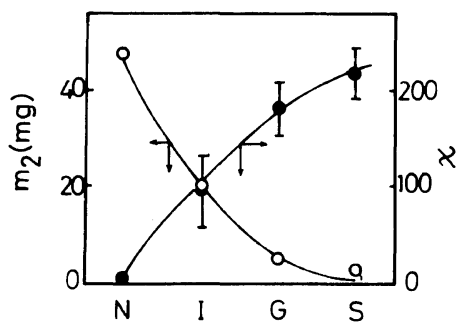

Figure 5. Correlation between anticoagulant activity $A_{\mathrm{Lw}}$, measured by the Lee-White method, and weight of thromb $m_{2}$ determined by the Imai method, or anticoagulant activity $\chi(\mathrm{IU} / \mathrm{mg})$ measured according to the Commentary of Japanese Pharmacopoeia.

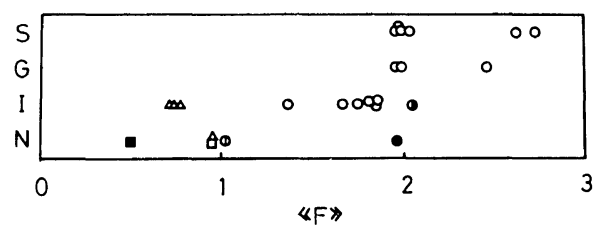

Figure 6. Effect of total degree of substitution $\langle F\rangle$ of $\mathrm{NaCS}$ on anticoagulant activity $A_{\mathrm{Lw}}: \bigcirc, \mathrm{CS}$ series; (1), CSD;, DSH; $\triangle$, HB series;, CS-A; $\square$, HBSD; $\square$, $\mathrm{HBH}$.

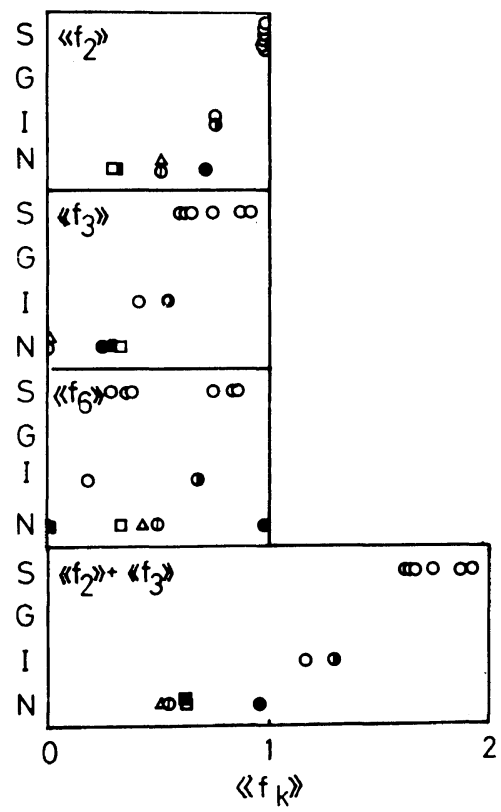

Figure 7. Effect of $\left\langle\mathrm{f}_{2}\right\rangle,\left\langle\mathrm{f}_{3}\right\rangle,\left\langle\mathrm{f}_{6}\right\rangle$ and $\left\langle\mathrm{f}_{2}\right\rangle+\left\langle\mathrm{f}_{3}\right\rangle$ of $\mathrm{NaCS}$ on anticoagulant activity $A_{\mathrm{LW}}$. Symbols have the same meaning as those in Figure 6.

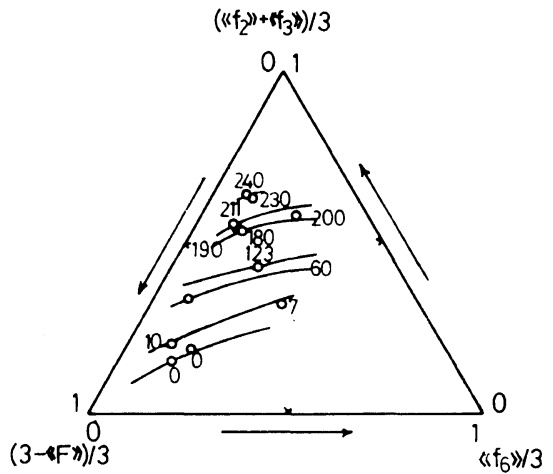

Figure 8. Dependence of anticoagulant activity $\chi$ of $\operatorname{NaCS}$ on $\left(\left\langle\mathrm{f}_{2}\right\rangle+\left\langle\mathrm{f}_{3}\right\rangle\right) / 3,\left\langle\mathrm{f}_{6}\right\rangle / 3$ and $(3-\langle\mathrm{F}\rangle) / 3:\left\langle\mathrm{f}_{k}\right\rangle$ is the distribution of sulfate groups on the carbon. position $k$ in glucopyranose units. $\chi$ is given on each curve. Open marks, experimental data.

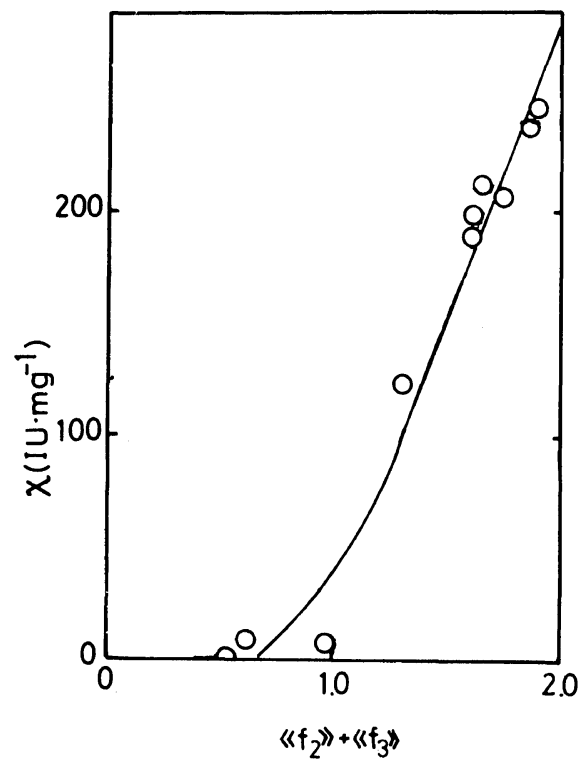

Figure 9. Effect of the sum of the substitution degree at carbon positions 2 and $3,\left\langle\mathrm{f}_{2}\right\rangle+\left\langle\left\langle\mathrm{f}_{3}\right\rangle\right.$, on anticoagulant activity $\chi$.

predominantly governed by $\left(\left\langle\mathrm{f}_{2}\right\rangle\right\rangle+\left\langle\left\langle\mathrm{f}_{3}\right\rangle\right) / 3$, being larger for larger $\left(\left\langle\mathrm{f}_{2}\right\rangle+\left\langle\left\langle\mathrm{f}_{3}\right\rangle\right) / 3\right.$ at constant $\left\langle\mathrm{f}_{6}\right\rangle / 3$ or $\langle\mathrm{F}\rangle / 3$. If compared at a fixed $\left(\left\langle\mathrm{f}_{2}\right\rangle+\left\langle\left\langle\mathrm{f}_{3}\right\rangle\right) / 3, \chi\right.$ is larger for smaller $\left\langle\mathrm{f}_{6}\right\rangle / 3$ and $\langle\mathrm{F}\rangle / 3$. However, $\left\langle\left\langle\mathrm{f}_{6}\right\rangle / 3\right.$ and $\langle\mathrm{F}\rangle / 3$ are not very sensitive to $\chi$. Figure 9 indicates that $\chi$ corresponding to the maximum value of $\left(\left\langle\mathrm{f}_{2}\right\rangle+\left\langle\left\langle\mathrm{f}_{3}\right\rangle\right)(=2)\right.$ is 285 .

These results seem to be the first demonstration 
of a close correlation between physiological activity and chemical structure of macromolecules. To obtain a polymer drug with desirable physiological activity, it is important to make a precise design of the molecular structure. For many years, it has been known that the anticoagulant activity of naturallyoccurring heparin varies significantly with its source and preparative method. The cause of this phenomenon can be understood if the distribution of

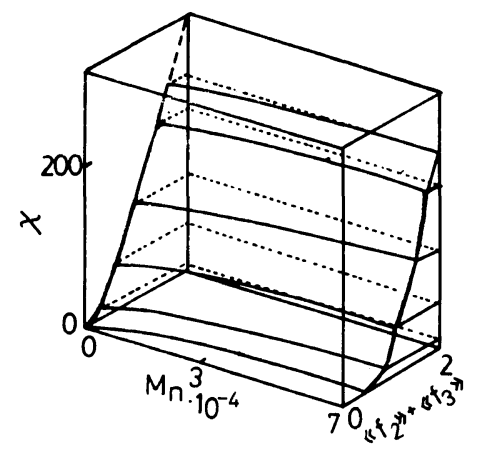

Figure 10. Anticoagulant activity $\chi$ plotted against number-average molecular weight $M_{n}$ and the sum of the substitution degree at carbon positions 2 and 3 , $\left\langle\mathrm{f}_{2}\right\rangle+\left\langle\left\langle\mathrm{f}_{3}\right\rangle\right.$.

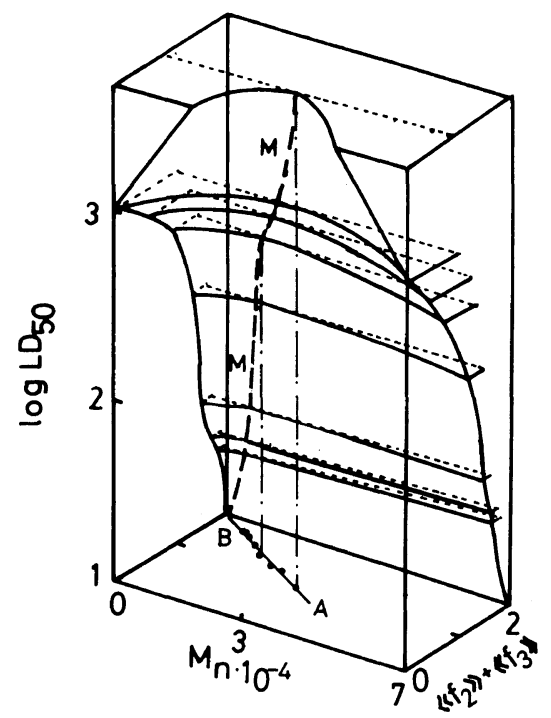

Figure 11. Plots of acute toxicity $\mathbf{L D}_{50}$ of $\mathrm{NaCS}$ against $M_{n}$ and $\left\langle\mathrm{f}_{2}\right\rangle+\left\langle\left\langle\mathrm{f}_{3}\right\rangle\right.$. The line $M$ represents maximum values of $\left\langle\mathrm{f}_{2}\right\rangle+\left\langle\mathrm{f}_{3}\right\rangle$ for a given $\mathrm{LD}_{50}$ (hereafter denoted by $\left(\left\langle\mathrm{f}_{2}\right\rangle+\left\langle\left\langle\mathrm{f}_{3}\right\rangle\right)_{m} . M_{n}\right.$ on the line $M$ is denoted by $\left(M_{n}\right)_{m}$. AB denotes the projection of the line $M$ on the plane $\mathrm{LD}_{50}=0$. chemical structure in heparin is fully elucidated, as in the case of $\mathrm{NaCS}$.

Figures 10 and 11 illustrate $\chi$ and $\mathrm{LD}_{50}$ as functions of $\left\langle\mathrm{f}_{2}\right\rangle+\left\langle\mathrm{f}_{3}\right\rangle$ and $M_{n}$. The full lines represent the contours of the same $\chi$ (Figure 10) and $\mathrm{LD}_{50}$ (Figure 11). Larger $\left\langle\mathrm{f}_{2}\right\rangle+\left\langle\mathrm{f}_{3}\right\rangle$ gives larger $\chi$ when compared at the same $M_{n}$. For fixed $\left\langle\mathrm{f}_{2}\right\rangle+\left\langle\mathrm{f}_{3}\right\rangle, \chi$ tends to increase with a decrease in $M_{n}$. From the anticoagulant activity point of view, it is highly desirable to prepare NaCS with higher $\left\langle\mathrm{f}_{2}\right\rangle+\left\langle\left\langle\mathrm{f}_{3}\right\rangle\right.$ and lower $M_{n}$. This suggests that the mobility of $\mathrm{NaCS}$ in solution is a minor factor in its anticoagulant activity. In Figure 11, the contours of the same $\mathrm{LD}_{50}$ are convex against $M_{n}$ axis, and the broken line $\mathrm{M}$ connecting the points for maximum

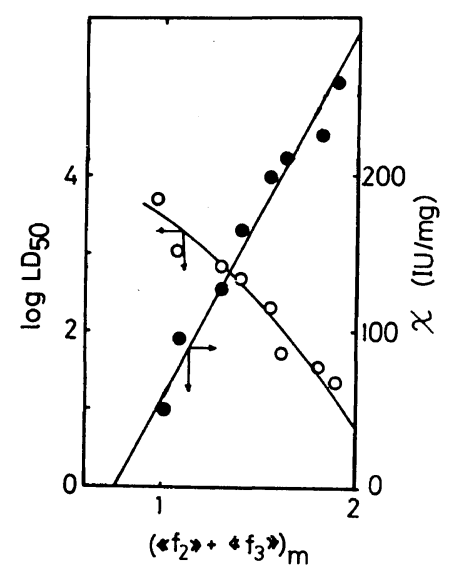

Figure 12. Relation between $\left(\left\langle\mathrm{f}_{2}\right\rangle+\left\langle\left\langle\mathrm{f}_{3}\right\rangle\right)_{m}\right.$ and acute toxicity $\mathrm{LD}_{50}$ or anticoagulant activity.

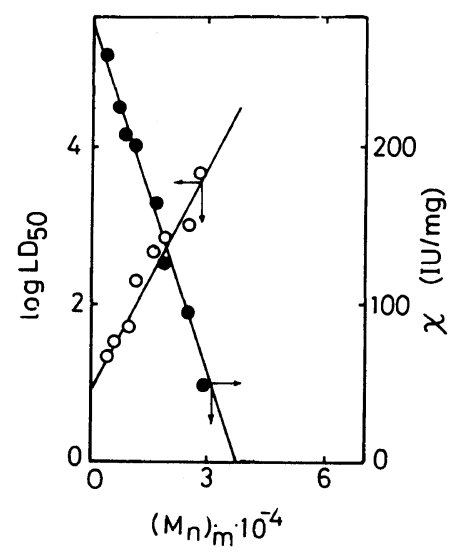

Figure 13. Relation between $\left(M_{n}\right)_{m}$ and acute toxicity $\mathrm{LD}_{50}$ or anticoagulant activity $\chi$. 
Table II. Existence ratios of coagulation factors and degree of inhibitory action $100\left(Y_{0}-Y\right) / Y_{0}$ of anticoagulants

\begin{tabular}{|c|c|c|c|c|c|c|c|c|c|c|c|c|c|c|c|c|c|}
\hline \multirow[t]{2}{*}{$\begin{array}{l}\text { Anticoagu- } \\
\text { lants }\end{array}$} & \multirow{2}{*}{$\frac{\chi}{\mathrm{IU} / \mathrm{mg}}$} & \multicolumn{8}{|c|}{$\frac{Y}{\%}$} & \multicolumn{8}{|c|}{$\frac{100\left(Y_{0}-Y\right) / Y_{0}}{\%}$} \\
\hline & & II & $\mathrm{V}$ & VII & $\mathrm{X}$ & VIII & IX & XI & XII & II & $\mathrm{V}$ & VII & $\mathrm{X}$ & VIII & IX & XI & XII \\
\hline Heparin $^{\mathbf{a}}$ & $188-228$ & 41.5 & 102.5 & 2.4 & 42.2 & 90.6 & 0.0 & 86.6 & 3.5 & 57.5 & 24.2 & 97.6 & 64.8 & 30.1 & 100 & 27.7 & 99.1 \\
\hline CS-12 & $200-222$ & 31.6 & 112.2 & 2.2 & 38.9 & 26.3 & 20.8 & 78.4 & 51.5 & 68.4 & 16.9 & 97.8 & 67.5 & 80.0 & 76.0 & 34.6 & 85.6 \\
\hline CSD & $117-130$ & - & 112.2 & 2.4 & 34.1 & 63.0 & 24.4 & 103.4 & 157.0 & - & 16.9 & 97.6 & 71.5 & 51.9 & 71.9 & 13.7 & 56.0 \\
\hline CS-5 & $51-61$ & 30.1 & 94.7 & 2.4 & 47.3 & 115.5 & 78.3 & 119.8 & 483.9 & 69.2 & 29.8 & 97.6 & 60.5 & 11.9 & 9.8 & 8.0 & 35.6 \\
\hline $\mathrm{HBH}$ & $12-15$ & - & 85.1 & 2.3 & 47.3 & 115.5 & 62.8 & - & - & - & 37.0 & 97.7 & 60.5 & 11.9 & 27.7 & - & - \\
\hline HBSD & $10-11$ & - & 78.7 & - & - & - & - & - & - & - & 41.6 & - & 一 & - & - & - & - \\
\hline CS-A & $7-8$ & - & 107.1 & 2.4 & - & 120.8 & 68.8 & 111.3 & - & - & 20.6 & 97.6 & - & 7.9 & 20.8 & 7.1 & - \\
\hline HB-1 & 0 & 44.1 & 89.1 & 2.3 & $50: 0$ & - & - & - & - & 54.9 & 34.0 & 97.7 & 57.8 & - & - & - & - \\
\hline None $^{b}$ & - & 97.7 & 134.2 & 100 & 119.8 & 131.1 & 86.8 & 119.8 & 356.7 & - & - & - & - & - & - & - & - \\
\hline
\end{tabular}

a Purified heparin.

b Denotes $Y_{0}$ for each coagulation factor in sample plasma. 
$\left\langle\mathrm{f}_{2}\right\rangle+\left\langle\mathrm{f}_{3}\right\rangle$ at a given $\mathrm{LD}_{50}$ shifts to lower $M_{n}$ with a decrease in $\mathrm{LD}_{50}$, as can be seen from the projected curve $\mathrm{AB}$ on the plane of $\mathrm{LD}_{50}=0$. $\left\langle\mathrm{f}_{2}\right\rangle+\left\langle\mathrm{f}_{3}\right\rangle$ and $M_{n}$ at a point on the line $\mathrm{M}$ are denoted hereafter by $\left(\left\langle\mathrm{f}_{2}\right\rangle\right\rangle+\left\langle\left\langle\mathrm{f}_{3}\right\rangle\right)_{m}$ and $\left(M_{n}\right)_{m}$, respectively. When compared at the same $M_{n}, \mathrm{LD}_{50}$ decreases as $\left\langle\mathrm{f}_{2}\right\rangle+\left\langle\left\langle\mathrm{f}_{3}\right\rangle\right.$ increases. This condition causes trouble in obtaining $\mathrm{NaCS}$ with a high anticoagulant activity and a desirable $\mathrm{LD}_{50}$. The line $M$ represents maximum $\left\langle\mathrm{f}_{2}\right\rangle+\left\langle\left\langle\mathrm{f}_{3}\right\rangle\right.$ for $\mathrm{a}$ $\mathrm{NaCS}$ sample, hence the maximum anticoagulant activity for a given $\mathbf{L D}_{50}$. This line gives $\left\langle\mathrm{f}_{2}\right\rangle+\left\langle\mathrm{f}_{3}\right\rangle=2$ at $M_{n}=0$ (i.e., the fully substituted glucose sulfate).

Figures 12 and 13 show the effects of $\left(\left\langle\mathrm{f}_{2}\right\rangle+\left\langle\left\langle\mathrm{f}_{3}\right\rangle\right)_{m}\right.$ and $\left(M_{n}\right)_{m}$ on $\chi$ and $\mathrm{LD}_{50}$. From these figures, $\chi$ and $\mathrm{LD}_{50}$ at $\left\langle\mathrm{f}_{2}\right\rangle+\left\langle\mathrm{f}_{3}\right\rangle=2$ and at $M_{n}=0$ are estimated to be 305 and 8 , respectively. The former is in very good agreement with the value 285 estimated from Figure 9. At $\left\langle\mathrm{f}_{2}\right\rangle+\left\langle\mathrm{f}_{3}\right\rangle=0$ and $M_{n}=3.7 \times 10^{4}, \mathrm{LD}_{50}$ is expected to be 10000 , implying that such a NaCS sample is practically non-toxic but its $A_{\mathrm{Lw}}$ is of the $\mathrm{N}$ grade. No NaCS sample having an anticoagulant activity and acute toxicity equivalent to heparin $\left(\chi=152\right.$ and $\mathrm{LD}_{50}=$ 1200 ) can be anticipated, since these figures indicate that $\mathrm{NaCS}$ has an acute toxicity higher than heparin which exists in the artery wall of animals and human beings. The next to the best molecular and structural parameters of $\mathrm{NaCS}$ as anticoagulants can be estimated from Figures 12 and 13. Given $\mathrm{LD}_{50}$ at the level of heparin, NaCS with $M_{n}=$ $1.65 \times 10^{4}$ and $\left\langle\mathrm{f}_{2}\right\rangle+\left\langle\mathrm{f}_{3}\right\rangle=1.23$ (so that $\chi$ is 120 ) is acceptable. If $\chi$ is desired to be $152, M_{n}=2.4 \times 10^{4}$ and $\left\langle\mathrm{f}_{2}\right\rangle+\left\langle\left\langle\mathrm{f}_{3}\right\rangle=1.40\right.$ are relevant and give $L_{50}=320-460$. It should be noted here that the oral $\mathrm{LD}_{50}$ of $\mathrm{NaCS}$ for a rat was found to be larger than $15000 \mathrm{mg} \mathrm{kg}^{-1}$, and hence presents no toxic problem.

The above discussion is concerned entirely with whole blood. Since 12 blood coagulation factors are known, it should be of interest to determine how and what extent $\mathrm{NaCS}$ acts on these factors. Table II shows the existence ratio $Y(\%)$ of each coagulation factor and the anticoagulating ability $100\left(Y_{0}-Y\right) / Y_{0}$, as well as $\chi$ of the anticoagulants. Figure 14 shows $100\left(Y_{0}-Y\right) / Y_{0}$ for various coagulation factors plotted against $\chi$. From this figure, the coagulation factors may be classified into two categories:
(1) $100\left(Y_{0}-Y\right) / Y_{0}$ varies with $\chi$ : Factors V, VIII, IX, XI, and XII. Factors VIII, XI, and XII increase in linear proportion to $\chi$ and factor IX increases rapidly with $\chi$, approaching some asymptotic value. In contrast, factor $\mathrm{V}$ has a tendency to decrease gradually with increasing $\chi$.

(2) $100\left(Y_{0}-Y\right) / Y_{0}$ is almost independent of $\chi$ and decreases in the following order: factor VII $>$ factor $\mathrm{X}>$ factor II.

We cannot explain why some factors are independent of $\chi$ and others decrease with $\chi$ and, in

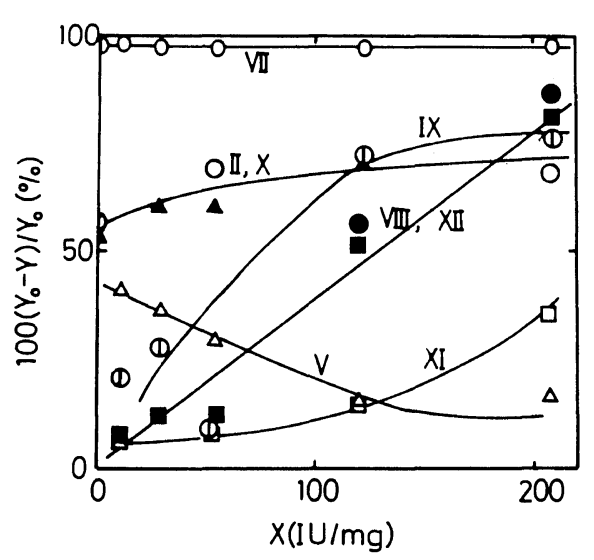

Figure 14. Correlation between $100\left(Y_{0}-Y\right) / Y_{0}$ of various coagulation factors and anticoagulant activity of NaCS: Factor II, O; V, $\triangle$; VII, O; VIII, $\mathbf{\square}$; IX, (1); $\mathrm{X}, \Delta ; \mathrm{XI}, \square ; \mathrm{XII}$,

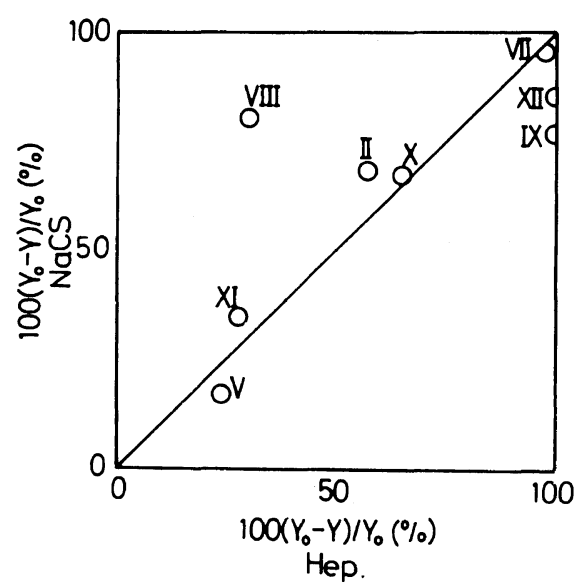

Figure 15. Comparison of $100\left(Y_{0}-Y\right) / Y_{0}$ between $\mathrm{NaCS}$ (CS-12) and heparin: Roman numbers indicate the order of coagulation factors. Symbols are the same as those in Figure 14. 
particular, why factor VII vanished by addition of $\mathrm{NaCS}$, even if the polymer has no anticoagulant activity $(\chi=0)$, as in the case of sample HB-1.

The inhibitory action of $\mathrm{NaCS}$ is compared with that of heparin in Figure 15, where $100\left(Y_{0}-Y\right) / Y_{0}$ for the two polymers having almost the same $\chi$ value $(190 \pm 10)$ are plotted on the horizontal and vertical axes. Except for factor VIII, the action of

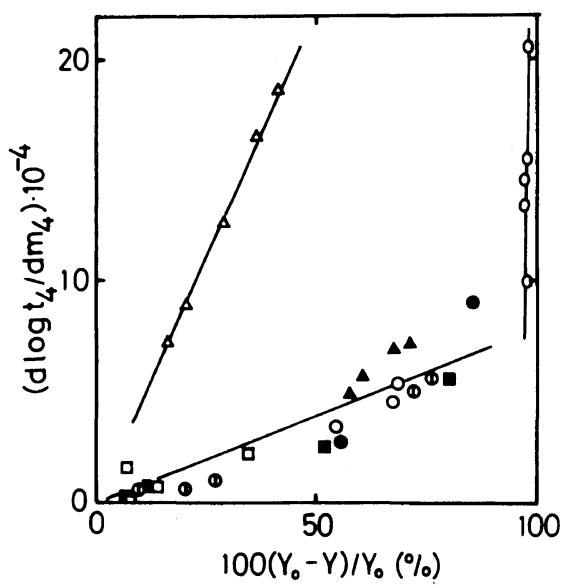

Figure 16. Plots of $\mathrm{d} \log t_{4} / \mathrm{d} m_{4}$ against $100\left(Y_{0}-Y\right) / Y_{0}$ for various coagulation factors. Symbols are the same as those in Figure 14.

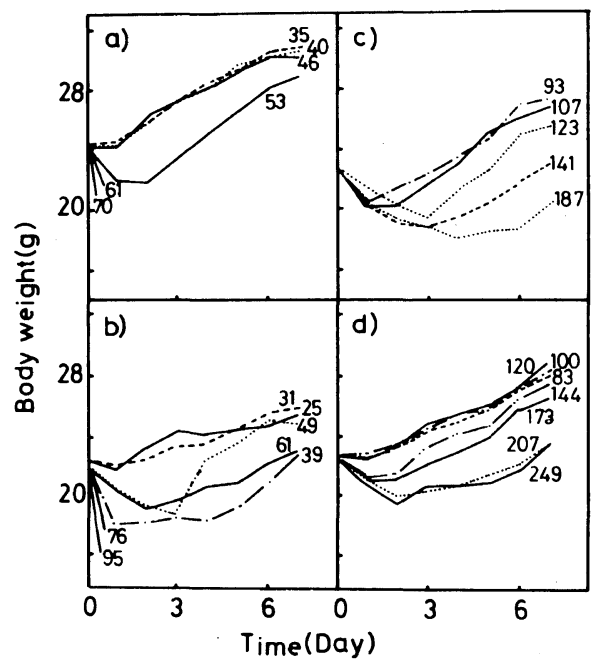

Figure 17. Changes in body-weight of rats with time following injection of a NaCS solution: a) Sample CS11, b) Sample CS-4, c) Sample CS-12, and d) Sample CS6. Numbers on the curves indicate the injected amounts of $\mathrm{NaCS}$ in $\mathrm{mg} \mathrm{kg}^{-1}$.
$\mathrm{NaCS}$ on all coagulation factors is the same as that of heparin. NaCS inhibits the action of factor VIII (antithermophilic globulin) much more effectively than heparin. Therefore, it may be concluded that $\mathrm{NaCS}$ acts mainly on factor VIII and partly on factor IX (plasma thromboplastin component); the latter was found to be suppressed by heparin. ${ }^{19}$

Figure 16 illustrates the relation between $\mathrm{d} \log t_{4} /$ $\mathrm{d} m_{4}$ and $100\left(Y_{0}-Y\right) / Y_{0}$. Except for factor VII, a straight line passing the origin is obtained, although factor $\mathrm{V}$ is expected to depend more strongly on concentration than the other factors.

Figures 17(a)-(d) illustrate time changes in the body-weight of rats after saline solutions of $\mathrm{NaCS}$ samples with four different $\mathrm{LD}_{50}(43,53.6,63.8$ and 184.5) were injected. In obtaining these data, we prepared polymer solutions of different concentrations and injected the same volume of each solution into a rat. The numbers on the lines denote the injected $\mathrm{NaCS}$ expressed in $\mathrm{mg} \mathrm{kg}^{-1}$. For all $\mathrm{NaCS}$ samples, when the dose amount was less than the $\mathrm{LD}_{50}$ of a given $\mathrm{NaCS}$, there was progressive

(a)

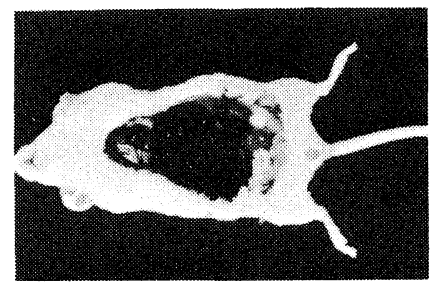

(b)

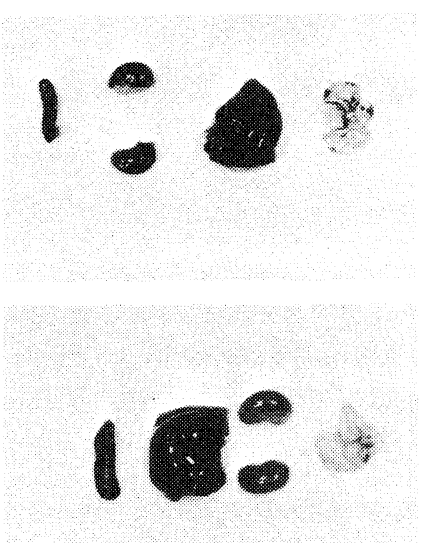

Figure 18. Typical anotomical photographs of live rats (a) and those of the intestinal organs of live (b) and dead (c) rats. 
body-weight increase. When the dose amount was more than the $\mathrm{LD}_{50}$, body-weight decreased at least initially, passed a minimum, and then increased monotonically. If the body-weight loss ratio $\left(B_{\mathrm{wL}}\right)$ is defined as the maximum body-weight loss $M(\mathrm{~g})$ divided by the dose amount $m\left(\mathrm{mg} \mathrm{kg}^{-1}\right)$ and the $\mathrm{LD}_{50}$ of a given $\mathrm{NaCS}$, that is, $B_{\mathrm{wL}}=$ $M / m \cdot \mathrm{LD}_{50}$, then $B_{\mathrm{WL}}$ for all samples comes close to 0.08 . This indicates that body-weight loss in rats is determined primarily by the dose amount and the $\mathrm{LD}_{50}$ of the NaCS sample used.

General symptoms of rats after injection of $\mathrm{NaCS}$ were occasional staggering motion and depression of breathing. Some rats lay down and often fell into a fit of convulsions. However, these symptoms disappeared one to four days after the injection. The dead rats showed bleeding from nose and mouth. The bleeding seemed greater for larger volumes of injected $\mathrm{NaCS}$ solution. In live rats, anemia was found.

Figure 18 shows some typical anatomical photographs of live rats (a) and the intestinal organs of live (b) and dead (c) rats after injection of NaCS. In the dead rats, the liver showed anemia and the lung, congestion and bloodshots. These abnormal symptoms were conspicuous in rats to which larger amounts of $\mathrm{NaCS}$ had been administered. In the live rats, no anormalous symptoms were found.

It is generally known that muco-polysaccharide derivatives including heparin exhibit a wide variety of pharmaceutical activities. In this work, we have shown that the most important factor governing the anticoagulant activity of $\mathrm{NaCS}$ is not the average molecular weight but the average chemical structure. It would be fruitful to carry out further research on the yet poorly understood physiological activities of polymer drugs by the methods presented in this study.

Acknowledgement. The authors should like to express their sincere appreciation to Dr. Kimihiko
Sato of the Sato-Takaida Hospital, Higashi Osaka City, for his assistance in the anticoagulant activity evaluation and to Mr. Masayuki Mizuno of the Department of Development of Medical Supplies, Ashashi Chemical Industry Company Ltd., for his kind experimental help in evaluating $\mathrm{LD}_{50}$ and the anatomical surveys.

\section{REFERENCES}

1. S. Bergström, Naturwissenshaften, 25, 706 (1935).

2. T. Astrup, I. Galsmar, and M. Volkert, Acta Physiol. Scand., 8, 215 (1944).

3. P. Karrer, H. Köenig, and E. Usteri, Helv. Chim. Acta, 26, 1296 (1943).

4. J. Astrup and J. Piper, Acta Physiol. Scand., 9, 351 (1945).

5. J. Piper, Acta Physiol. Scand., 9, 28 (1945).

6. J. Piper, "Farmakologiske Undersögelser över Syntetiske Heparin-lignande Stoffer," (Disp.) Copenhagen, 1945.

7. J. Felling and C. E. Wiley, Arch. Biochem. Biophys., 85, 313 (1959).

8. A. M. Rothschild, J. Pharmac. Chemother., 33, 501 (1968).

9. A. M. Rothschild and A. Castania, J. Pharm. Pharmac., 20, 77 (1968).

10. J. Kiss, "Chemical Structure of Heparin," in "Heparin," K. Thomas, ed., Academic Press, London, 1976, p 9.

11. K. Kamide and K. Okajima, Polym. J., 13, 163 (1981).

12. K. Kishino, T. Kawai, T. Nose, M. Saito, and K. Kamide, Eur. Polym. J., 17, 623 (1981).

13. R. Schweiger, Carbohydr. Res., 21, 219 (1972).

14. I. Kanai and M. Kanai eds., in "Rinsho Kensaho Teiyo,". Kinbara Publishing Co., Tokyo, 1975, Chapter VI, p 85.

15. Y. Imai and Y. Nose, J. Biomed. Mater. Res., 6, 165 (1972).

16. Commentary of Japanese Pharmacopoeia, No. C1235-C1242, Nankodo, Tokyo (1965).

17. Technical Sheet, No. CO 15-4313, CO 16-4828E, CO 17-4379, CO 18-4378E, 1978 (Diagnostic Inc., USA).

18. B. Probit, Ann. Appl. Biol., 22, 134 (1934).

19. M. A. Lyapina, Frigiologia Chelveka, 4, 295 (1978). 